\title{
The reliability of height and height velocity in the assessment of growth (the Wessex Growth Study)
}

\author{
L D Voss, T J Wilkin, B J R Bailey, P R Betts
}

\begin{abstract}
Both biochemical and auxological measurements can be used to assess growth. Quality control is routinely reported in laboratory studies, but the reproducibility of height measurements, and the height velocity data derived from them, is seldom considered. We have previously established our error and in this report we examine its implications for the screening of short children and subsequent monitoring of their growth. The $95 \%$ confidence interval for height for a 5 year old observed to be on the 3rd centile for height, spanned the 2nd-4th centile. However, the confidence interval for a 12 month height velocity appropriate to such a child spanned the 8th-52nd centiles, the lower limit pathological and the upper more than satisfactory. A single height velocity even over 12 months therefore lacks the precision to provide a reliable index of current growth in short children. Furthermore, serial height velocity calculations on a cohort of $\mathbf{7 8}$ short normal children showed no significant correlation from year to year, suggesting that velocity is also unable to predict future growth. Although the proportion of this cohort of short children lying beneath the 25 th centile for velocity remained constant from year to year, the identity of the individuals comprising that proportion changed, a phenomenon which could be largely accounted for by the random error associated with height velocity. Our data suggest that, by the time a trend in abnormal velocity is reliably established, a deviation from the height centiles is clearly evident. Although velocity charts are attractive in concept, they seem to be no more discriminating than height charts in practice, and may be clinically deceptive unless interpreted with great care.
\end{abstract}

Poor growth in childhood may be a sign of poor health and deserves careful attention. ${ }^{12}$ In the community short stature is usually identified by cross sectional screening of particular age groups, for example school entrants, and we have already drawn attention to the errors in screening associated with secular trend and poor calibration of equipment used. ${ }^{3-5}$

Clinically, difficulties have arisen with the interpretation of stress tests for growth hormone release, and paediatricians have reemphasised the value of auxological data to identify children who might benefit from growth hormone treatment. ${ }^{6-8}$

The measurement of height, like any other, is subject to error, but the size and implications of this error are seldom considered. ${ }^{9}$ Moreover, height velocity has been promoted as a better means of expressing growth than height alone, ${ }^{1} 10$ " 11 although it seems a priori unlikely that velocity, which is derived from height measurements, can be inherently more informative.

If velocity is to be used as an auxological tool, it should be recognised that there are very few data available on its reproducibility and the confidence with which paediatricians can use it for making clinical decisions. We have established our error of height measurement, and in this paper we examine its implications for the screening of short children and the subsequent monitoring of their growth.

\section{Patients and methods}

The clinical component of this study is based on the growth data of two cohorts of short children from the Wessex Growth Study, on or below the 3rd centile for height according to Tanner and Whitehouse standards, ${ }^{12}{ }^{13}$ who entered first school at the age of 5 during the school years 1985/6 and 1986/7, and who have been measured annually since by a single trained auxologist (LDV) using an electronic stadiometer (Holtain). Ethnic minorities, children with obvious organic disease, and children with psychosocial problems, identified by a behaviour questionnaire, were excluded from this study. Two years' height data are available for the remaining 78 short normal children (all of whom had normal thyroid function tests and normal concentrations of haemoglobin, creatinine, urea, and electrolytes) and 65 case controls of normal height (10th-90th centile) and same sex, age, and class at school. The mean height SD scores of the short children and their controls were $-2.26(0.32)$ and $0.47(0.32)$ respectively.

Our error of measurement, expressed as the SD of a single height measurement (SDshm), was established as $0.25 \mathrm{~cm}$ from a series of calibration trials, the method for which has been reported previously. ${ }^{5}$ The $95 \%$ confidence limits of a single height measurement $( \pm 2 \times$ SDshm) were therefore $\pm 0.5 \mathrm{~cm}$. The corresponding $95 \%$ confidence limits for expressing a 12 month height velocity were deduced from the formula: $\pm 2($ SDshm) $\sqrt{2} \mathrm{~cm} /$ year, giving a value of $\pm 0.71 \mathrm{~cm} /$ year. We applied these confidence limits to the Tanner and Whitehouse height and velocity standards appropriate to children of small and average height, in order to establish the error associated with these measurements expressed as absolute height (velocity), height SD scores (velocity SD scores) and height centiles (velocity centiles). 


\section{Results}

CLINICAL OBSERVATIONS

Two consecutive 12 month measurements of height velocity data from the 78 short normal school entrants and their controls are shown in fig 1 . Both groups of children had a normal distribution of height velocity. Although the mean velocity of the short children was significantly lower than that of their controls $(p<0.001)$ on both occasions, there was considerable overlap between them so that very few individual short children (six in year one and two in year two)
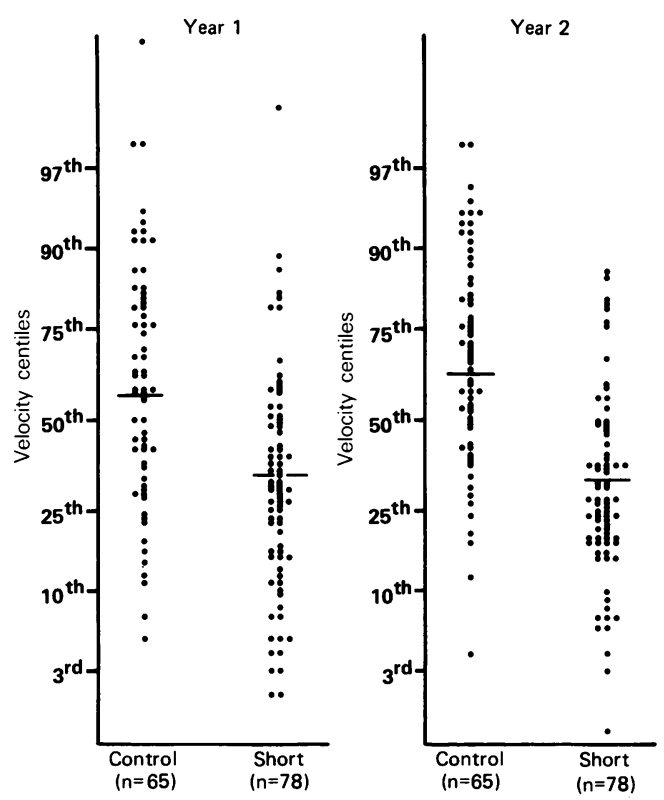

Figure 1 Scattergrams comparing the mean and distribution of 12 month height velocities of short normal $(\leqslant 3$ rd centile for height) and control (10th to 90 th centile for height) children 5 and 6 years of age. The data from two consecutive

12 month periods (year 1 and year 2) are shown.

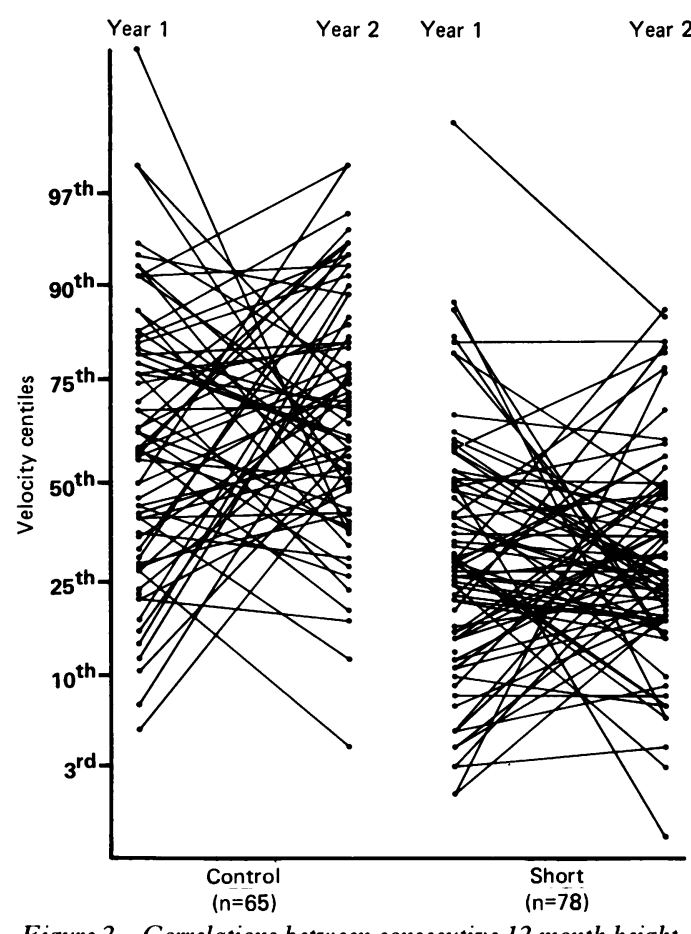

Figure 2 Correlations between consecutive 12 month heigh velocity values in the group of short normal children $(\leqslant 3 r d$ centile for height) and their controls (10th to 90th centile for height).
The $95 \%$ confidence limits for height and height velocity calculated for a short boy (3rd centile for height)

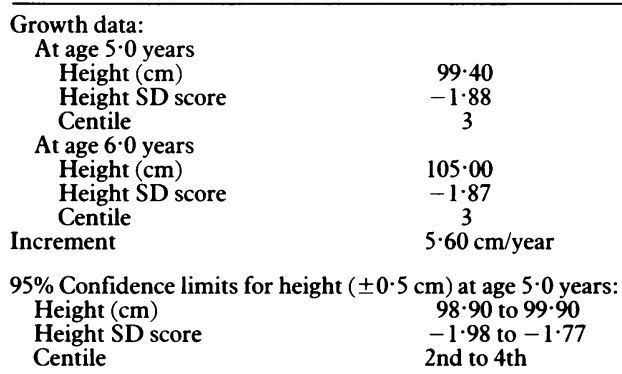

$95 \%$ Confidence limits for 12 month height velocity $( \pm 0 \cdot 71$ $\mathrm{cm} /$ year) between age 5.0 and 6.0 years:

$\begin{array}{ll}\text { Velocity cm/year } & 4.89 \text { to } 6.31 \\ \text { Velocity SD score } & -1.39 \text { to }+0.04 \\ \text { Centile } & 8 \text { th to } 52 \text { nd }\end{array}$

Growth standards from Tanner and Whitehouse. ${ }^{12} 13$

could be distinguished from controls on the basis of a single 12 month velocity. Averaging the two years, $41 \%$ of the short normal children had a velocity below the 25 th centile, and $15 \%$ a velocity below the 10 th centile.

There was no significant correlation between two consecutive 12 month velocity values in either the short children $(r=0.17, p>0.05)$ or their controls $(r=0.10, p>0.05)$ (fig 2). Although the proportion within each group which lay below the 25th and 10th centile respectively remained much the same from year to year, their identities largely changed. Only 14 $(18 \%)$ of the short children lay below the 25th velocity centile and three $(4 \%)$ below the 10 th centile on two consecutive occasions. The corresponding proportions of the control group were one $(1.5 \%)$ and none respectively.

IMPLICATIONS OF MEASUREMENT ERROR

The implications of a known error of measurement on the interpretation of height and height velocity data are adduced in the table. The range of centiles and SD scores crossed by the $95 \%$ confidence intervals for height and height velocity were calculated for a short boy, growing on the 3rd centile for height, between the ages of 5 and 6 years. The confidence intervals spanned a much wider range of velocity centiles (8th-52nd) than height centiles (2nd-4th) (fig 3).

The confidence intervals will be wider than those illustrated in cases where the error of measurement is greater than that reported here. The need for each auxologist to establish his or her error of measurement in the manner we have previously suggested is clear, ${ }^{5}$ and fig 4 provides a reference chart from which the confidence limits corresponding to a given SDshm can be determined.

Finally, fig 5 illustrates the true height and height velocity for a (theoretical) slow growing 5 year old child plotted over three years from school entry. Even were the child to retain the 10th centile for velocity throughout (an unlikely event), the precision is such that no individual value can be reliably distinguished from the 25 th centile. A trend to low velocity could be deduced from two successive values below the 25 th centile, ${ }^{11}$ but by this time (age 7 years), a 


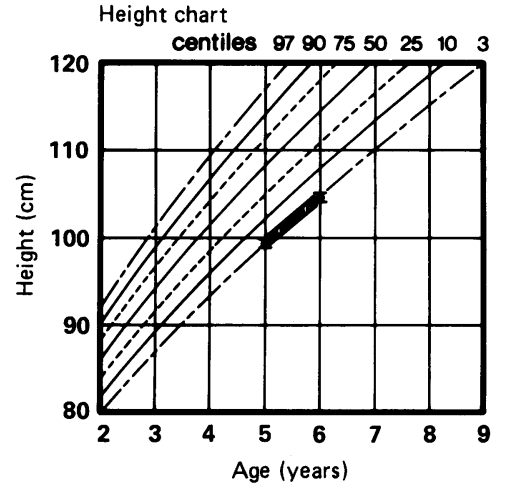

Velocity chart

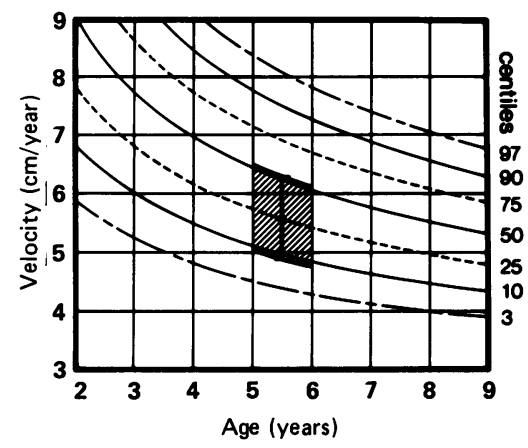

Figure 3 Tanner and Whitehouse height and velocity centiles on which have been plotted the $95 \%$ confidence intervals appropriate to a child between the age of 5 and 6 years, growing steadily along the 3rd centile for height, given a SD of a single height measurement of $0.25 \mathrm{~cm}$. departure from the 3rd centile for height is clearly evident. This is an idealised example. In practice, the wide fluctuations in successive velocity values illustrated in fig 2 are liable to occur, making the clinical interpretation of velocity data more difficult still.

\section{FOOTNOTE}

The $95 \%$ confidence interval for a change in velocity (acceleration/deceleration) can be derived from the formula $v_{2}-v_{1} \pm 2$ (SDshm) $\sqrt{6} \mathrm{~cm} /$ year/year. (The difference in velocity being based on three observations each one year apart.) Given an SDshm of $0.25 \mathrm{~cm}$, a change in velocity of at least $1.22 \mathrm{~cm} /$ year/year would therefore have to be recorded to represent reli-
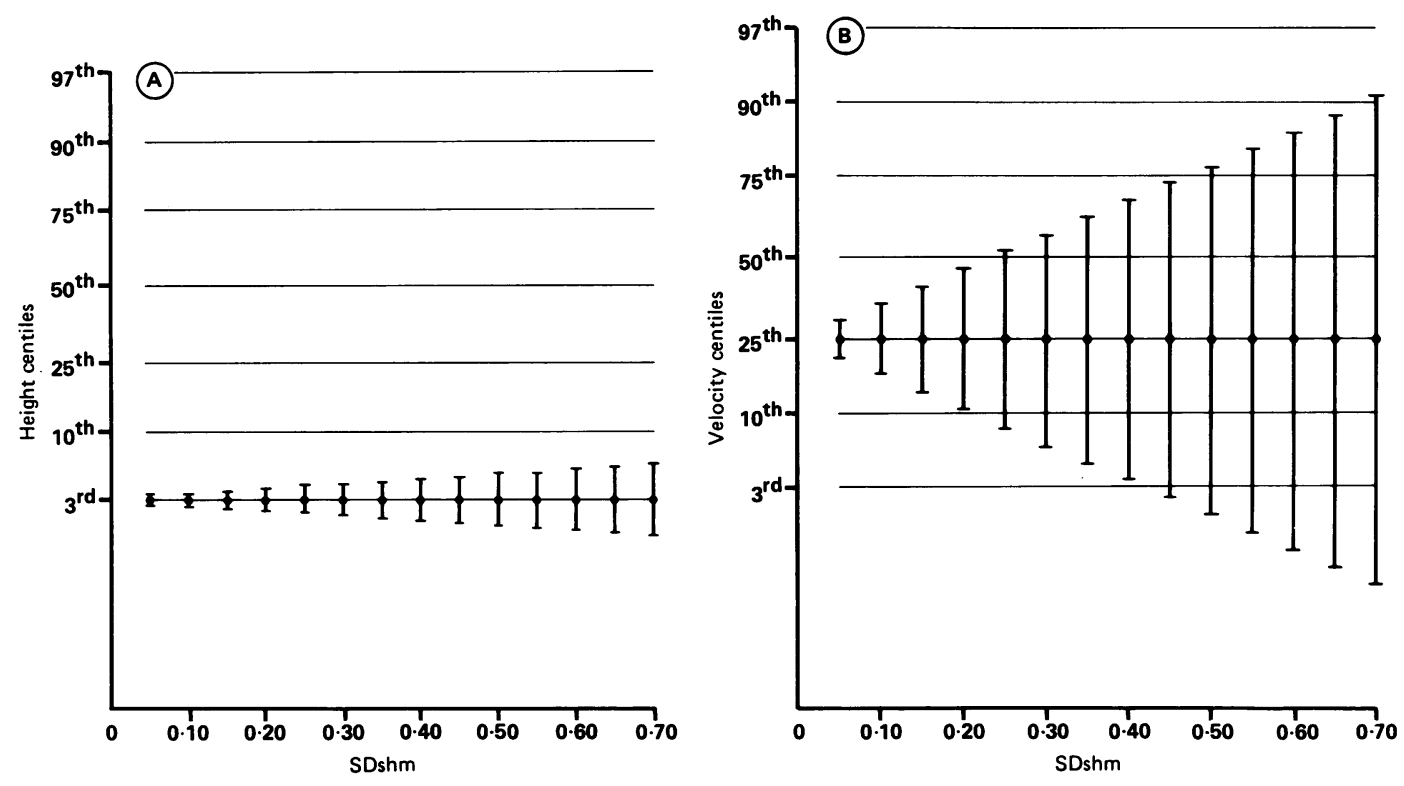

Figure 4 The $95 \%$ confidence intervals for $(A)$ height and $(B) 12$ month height velocity data which correspond to a range of $S D$ s for a single height measurement (SDshm) varying from 0.1 to $0.7 \mathrm{~cm}$. The illustration corresponds to a child of 5 years growing along the third centile for height (25th centile for velocity), but the data can be moved up and down the ordinate axis as appropriate.
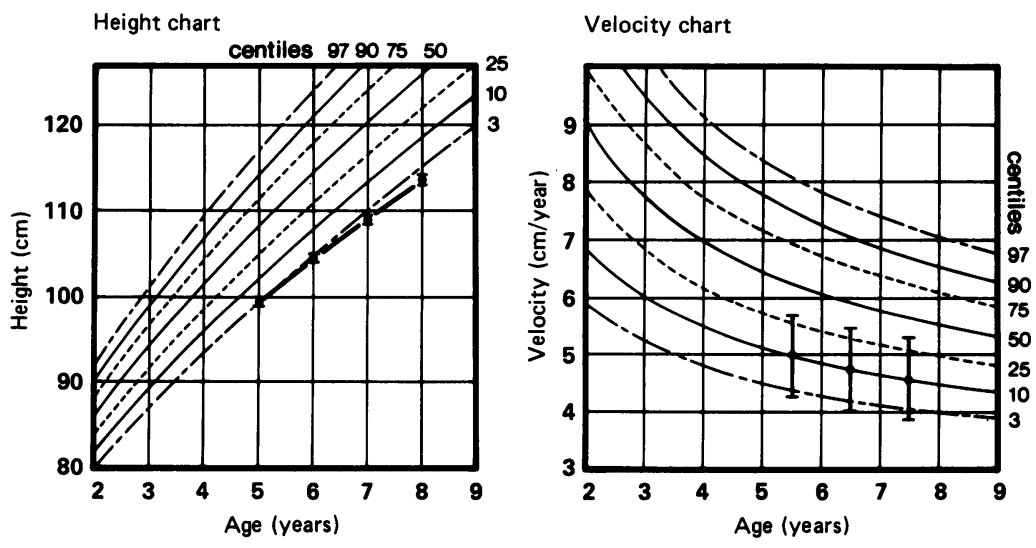

Figure 5 The appearance on height and velocity charts of the same data from a boy who was on the 3rd centile for height when first seen on his fifth birthday, and who grew slowly along the 10th centile for velocity over the next two years. The $95 \%$ confidence intervals for height and velocity corresponding to an SDshm of $0.25 \mathrm{~cm}$ are shown. ably a change. In practice, this means an acceleration to the 80th centile for a short 5 year old previously growing along the 25 th centile for velocity (that is, 3rd centile for height). Anything less could be random error, and the reporting of responses to height-promoting treatment should be qualified accordingly.

\section{Discussion}

This report does not deny height velocity a role in the auxological assessment of children, but it does seek to demonstrate that velocity is no more informative than height measurements alone, by establishing the confidence intervals appropriate to each. Because they are plotted on a magnified scale (0-24 cm rather than 45-195 $\mathrm{cm}$ as on a height chart), differences in velocity 
centile appear more convincing to the eye than the corresponding changes in height. But what appears to be gained in sensitivity is lost in reliability, as the error attaching to velocity is proportionately greater. The aim of this study was to explore the practical implications of the difference in reliability between height and velocity data.

In the Wessex Growth Study we were concerned to identify all school entrants in two health districts whose height lay below the 3rd centile. ${ }^{4}$ The error, expressed as the SD for a single height measurement, was $0.25 \mathrm{~cm}$ and gave $95 \%$ confidence limits which correspond to the 2 nd to 4 th centile. ${ }^{5}$ Our screening procedure, based as it was on a single height measurement, might therefore be expected to include a small number of children whose true height lay above the $3 \mathrm{rd}$ centile and to exclude a similar number from below.

For statistical reasons a short normal child who grows along the 3rd centile for height proceeds (on average) along the 25th centile for velocity. ${ }^{7}$ We have shown that, with an SDshm of $0.25 \mathrm{~cm}$, which is comparable with that quoted by Tanner ${ }^{14}$ and less than that shown by Prader et al, ${ }^{15}$ the confidence limits attaching to the 25 th centile for velocity span the 8 th-52nd centile. A velocity on the 8 th centile would be considered pathological, while a velocity on the 52nd centile, corresponding to a child of average height, would be more than adequate for a small child. These measurements were taken by an experienced auxologist; if height were measured with less precision on a less reliable instrument, the confidence limits would be greater to the degree shown in fig 4 . The error in velocity will be greater still if measurements are made less than 12 months apart. ${ }^{14}$ First, an interval of just four months, as has been recommended, ${ }^{16}$ would by itself triple the lengths of the confidence intervals illustrated. Second, measurements less than 12 months apart introduce seasonal variation. Diurnal variation will not influence the confidence intervals we have derived for height and height velocity, which were all based on measurements taken at the same time of day, but may be a further source of error when following up children in the clinic, where appointment times differ.

Although the distribution of the height velocities of the short children was predictably lower than that of the taller control children, there was such overlap that velocity could not be safely used to identify slow growers. On average, only about $5 \%$ of the short normal children on or below the 3rd centile for height had a growth velocity over 12 months below the range appropriate to the controls. Although the proportion of 'slow growers' defined by the 25 th velocity centile remained fairly constant from year to year, the individuals represented in this proportion changed, and there was no correlation in the growth velocities of individuals from year to year. Thus velocity in small children not only fails to reflect previous growth, ${ }^{4}$ but it also fails to predict future growth.

It has been suggested that two consecutive velocity values below the 25 th centile will iden- tify poor growth. ${ }^{11}$ This, however, is a calculation based on a model child drawn from the whole population. All normal children below the 3rd centile for height already average less than the 25 th centile for velocity (see above), so that the velocity centile appropriate to a diagnosis of poor growth is not fixed, and will vary with the height of the child. It may be possible to identify a significantly reduced velocity by applying trend statistics to the chance of lying on or below a particular centile on two or more occasions. ${ }^{11}$ However, two or preferably three measures of velocity would be needed to do this, implying at least two or possibly three years of observation. By this time, as we have shown, a fall on the height centile chart will be evident.

Our data show that in clinical practice a substantial proportion (18\%) of short normal children are observed to fall below the 25 th centile for velocity over two successive years compared with only $1 \%$ of taller controls. This, and the fact that a single 12 month velocity measurement can, through its imprecision, appear to be very low, should raise questions about the use of height velocity to determine the need for growth hormone treatment. ${ }^{6-8}$

From a pragmatic point of view there may be much to commend the concept of height velocity: it can be a useful tool for evaluating group data. For the assessment of individual children, however, velocity cannot be expressed with sufficient precision. It cannot identify poor growth more quickly and does not improve upon the height data from which it is derived. As we have pointed out previously, the variability is largely $(95 \%)$ due to the elasticity of children who are of no fixed height from one moment to the next. ${ }^{5}$ We conclude that there is considerable imprecision in the auxological assessment of growth, and we have been unable to demonstrate that either height or height velocity is superior to the other. When screening for poor growth or monitoring response to treatment in individuals we are inclined to recommend the height chart for its simplicity and (provided it is properly maintained) its relatively low risk of being misinterpreted. The fact remains, however, that making clinical decisions on the basis of short term growth data, whatever its form, may be misguided and there is a clear need for growth surveillance to start at an early age and to continue throughout childhood.

LDV is generously supported by Kabi Pharmacia UK Ltd and Sweden AB and TJW by the Wellcome Trust. We thank Ms BA
Peddle and Mrs J Mulligan for expert preparation of the manuscript.

1 Parkin JM. The short child. In: Brook CGD, ed. Clinical paediatric endocrinology. Oxford: Blackwell Scientific Publications, 1989:96-117.

2 Mahoney CP. Evaluating the child with short stature. Pediat Clin North Am 1987;34:825-49.

3 Voss LD, Wilkin TJ, Betts PR. Do we need new growth charts?' Lancet 1987;ii:447-8.

4 Voss LD, Walker J, Lunt H, Wilkin TJ, Betts PR. The Wessex growth study: first report. Acta Paediatr Scand [Suppl] 1989;349:65-72.

5 Voss LD, Bailey BJR, Cumming K, Wilkin TJ, Betts PR. The reliability of height measurement. Arch Dis Child 1990, 65:1340-44.

6 Brook CGD. Treatment of growth deficiency. Clin Endocrinol (Oxf) 1988;30:197-204. 
7 Hindmarsh PG, Brook CGD. Auxological and biochemical assessment of short stature. Acta Paediatr Scand [S uppl] 1988;343:73-6.

8 Milner RDG. Which children should have growth hormone therapy? Lancet 1986;i:483-5.

9 Cameron $\mathrm{N}$. The methods of auxological anthropometry. In: Falkner F, Tanner JM, eds. Human growth. 2nd Ed. New York: Plenum, 1986;3:3-46.

10 Tanner JM. Use and abuse of growth standards. In: Falkner F, Tanner JM, eds. Human growth. 2nd Ed. New York: Plenum, 1986;3:95-109.

11 Brook CGD, Hindmarsh PC, Healy MJR. A better way to detect growth failure. BMF 1986;293:1186.

12 Tanner JM, Whitehouse RH, Takaishi $M$. Standards from birth to maturity for height, weight, height velocity and weight velocity: British children 1965. Arch Dis Child 1966; 41:454-71.

13 Tanner JM, Whitehouse RH, Takaishi M. Standards from birth to maturity for height, weight, height velocity and weight velocity: British children 1965. Part II. Arch Dis Weight velocity: British

14 Tanner JM. Physical development. Br Med Bull 1986;42: 131-8.

15 Prader A, Largo RH, Molinari L, Issler C. Physical growth of Swiss children from birth to 20 years of age.. Helv Paediatr Acta 1988;Suppl 52:3-33.

16 Brook CGD, Hindmarsh PC. Tests for growth hormone secretion. Arch Dis Child 1991;66:85-7.

\section{Treating tuberculosis}

In 1983, 452 children were treated for tuberculosis in England and Wales. Four hundred and one of those had chest or lymph node disease and the treatment they were given was described in Archives in $1989 .{ }^{1}$ The results of treatment were good but concern was expressed about variability in drug dosages and treatment duration and about possible overusage of ethambutol and underusage of pyrazinamide. It was pointed out that there was little information about the use of short course chemotherapy in children.

The November 1990 issue of The Pediatric Infectious Disease Fournal contains two important reports of treatment trials and an excellent review article by Starke on pages 785-793.

The first of the trials was done in Papua New Guinea (Biddulph, pages 794-801) and is by far the largest yet reported, involving 639 children. For the first two months treatment was given in hospital using four drugs (rifampicin, isoniazid, pyrazinamide, and streptomycin) given daily and for the subsequent four months two drugs (rifampicin and isoniazid) were given twice a week under professional supervision. Despite high rates of default and non-compliance the results showed that six months' treatment is effective. Less than $2 \%$ of the children who completed a six month course relapsed and most of those had been irregular with their treatment.

The other trial was done in India (Kumar et al, pages 802-6) and involved 76 children. Thirty seven were given twice weekly treatment throughout, for the first two months with three drugs (rifampicin, isoniazid, and pyrazinamide) and for the next four months with two drugs (rifampicin and isoniazid). Thirty nine children were given daily treatment with three drugs for two months followed by twice weekly treatment with two drugs for four months. All treatment was given under professional supervision. Both treatment regimens were highly effective ( $>95 \%$ cure) and there were no relapses over two years of follow up. No serious side effects of the drugs requiring changes in treatment were encountered.

The advantages of three drug short (six month) course treatment include more rapid loss of infectivity in open disease, less time in which to be non-compliant, fewer resources required for treatment monitoring, and broader drug cover for resistant organisms. There seems to be no advantage in adding streptomycin. Extrapulmonary disease can be treated in the same manner but meningitis or bone and joint disease may need longer courses (see Starke, above).

The drug treatment of tuberculosis is a classical case for audit. There is no case to be made out for the therapeutic maverick. As recommendations change from time to time with the better use of old drugs and the introduction of new ones, my own practice is to ask a respiratory physician to look over my shoulder occasionally to make sure I'm still on the accepted track. The occasions on which it is useful to consult an adult orientated physician get fewer but this is still one of them. 